\title{
Parameterization of elastic stress sensitivity in shales
}

\author{
Marina Pervukhina ${ }^{1}$, Boris Gurevich ${ }^{2}$, Pavel Golodoniuc ${ }^{3}$, and David N. Dewhurst ${ }^{1}$
}

\begin{abstract}
Stress dependency and anisotropy of dynamic elastic properties of shales is important for a number of geophysical applications, including seismic interpretation, fluid identification, and 4D seismic monitoring. Using SayersKachanov formalism, we developed a new model for transversely isotropic (TI) media that describes stress sensitivity behavior of all five elastic coefficients using four physically meaningful parameters. The model is used to parameterize elastic properties of about 20 shales obtained from laboratory measurements and the literature. The four fitting parameters, namely, specific tangential compliance of a single crack, ratio of normal to tangential compliances, characteristic pressure, and crack orientation anisotropy parameter, show moderate to good correlations with the depth from which the shale was extracted. With increasing depth, the tangential compliance exponentially decreases. The crack orientation anisotropy parameter broadly increases with depth for most of the shales, indicating that cracks are getting more aligned in the bedding plane. The ratio of normal to shear compliance and characteristic pressure decreases with depth to $2500 \mathrm{~m}$ and then increases below this to $3600 \mathrm{~m}$. The suggested model allows us to evaluate the stress dependency of all five elastic compliances of a TI medium, even if only some of them are known. This may allow the reconstruction of the stress dependency of all five elastic compliances of a shale from log data, for example.
\end{abstract}

\section{INTRODUCTION}

Shales are the most common rock type encountered in sedimentary basins. Due to their strongly anisotropic properties, the presence of shales in the subsurface may cause significant errors in depth obtained from surface seismic data, in normal moveout correction, dip moveout correction, migration, and amplitude variation with offset analysis (e.g., Castagna et al., 1993; Banik, 1984; Sayers, 1999; Tsvankin et al., 2009). Seismic anisotropy of shales is caused by both intrinsic anisotropy of clay minerals that are abundant in shales and by preferred orientation of clay platelets (e.g., Hornby et al., 1994; Johnston and Christensen, 1995; Sayers, 1999).

Various studies have addressed the problem of prediction of shale anisotropy, which requires values of the five elastic stiffness coefficients required to describe a transversely isotropic (TI) medium and Thomsen's anisotropy parameters (Thomsen, 1986). Johnston and Christensen (1995) report a strong positive correlation between seismic anisotropy and so-called orientation indices that reflected clay mineral alignment. Johansen et al. (2004) study the effect of alignment of grain scale texture on seismic anisotropy and reflectivity of shales. Sayers (2005) related the clay platelet orientation distribution to the behavior of Thomsen's $\delta$ parameter, which is the only anisotropy parameter needed to interpret the small-offset amplitude variation with offset response. Using self-consistent approximation (SCA) effective media theory, Hornby et al. (1994) reconstruct the TI elastic tensor of the Greenhorn Shale on the basis of the knowledge of clay platelet orientation distribution and silt inclusion fraction, assuming knowledge of clay elastic properties. All these previous studies required knowledge of detailed microstructure and assumed that the anisotropic elastic moduli of individual clay minerals are known. However, the clay platelet orientation characteristics, which require thorough quantitative image analysis, can vary from sample to sample even for shale samples acquired just a few meters apart. In addition, elastic properties of clay minerals reported in the literature range in value by more than an order of magnitude (e.g., Aleksandrov and Ryzhova, 1961; Woeber et al., 1963; Han et al., 1986; Castagna et al., 1993; Hornby et al., 1994; Katahara, 1996; Vanorio et al., 2003; Ortega et al., 2007)

Manuscript received by the Editor 17 August 2010; revised manuscript received 23 December 2010; published online 23 May 2011.

${ }^{1}$ CSIRO Earth Science and Resource Engineering, ARRC, Kensington, Australia. E-mail: Marina.Pervukhina@csiro.au; David.Dewhurst@csiro.au.

${ }^{2}$ Curtin University, Department of Exploration Geophysics, Perth, Australia and CSIRO Earth Science and Resource Engineering, ARRC, Kensington, Australia. E-mail: B.Gurevich@curtin.edu.au.

${ }^{3}$ CSIRO Earth Science and Resource Engineering, ARRC, Kensington, Australia and Curtin University, Department of Exploration Geophysics, Perth, Australia. E-mail:Pavel.Golodoniuc@csiro.au.

(C) 2011 Society of Exploration Geophysicists. All rights reserved. 
and depend on a number of parameters, such as clay mineralogy, water content, and sample preservation.

Recently, Ulm and Abousleiman (2006) suggest a simple model that allows prediction of anisotropic elastic moduli of shales. This model implies that shale elastic properties are independent of clay mineralogy and allows prediction of elastic moduli on the basis of only two parameters, namely, silt fraction and clay packing density (CPD), a volumetric fraction of clay minerals in wet shale without silt inclusions. Using this model in combination with the SCA approach, Ulm and Abousleiman (2006) estimate elastic moduli of clay minerals from measurements of elastic properties of a number of shales. Bayuk et al. (2007) use the general singular approximation effective media approach to estimate the elasticity tensor of clay minerals from experimentally measured elastic properties of the Greenhorn Shale used in Hornby's study (Hornby et al., 1994). The approach of Ulm and Abousleiman (2006) and Bayuk et al. (2007) is further refined by Pervukhina et al. (2008a, 2008b), who suggested using a differential effective media approach as

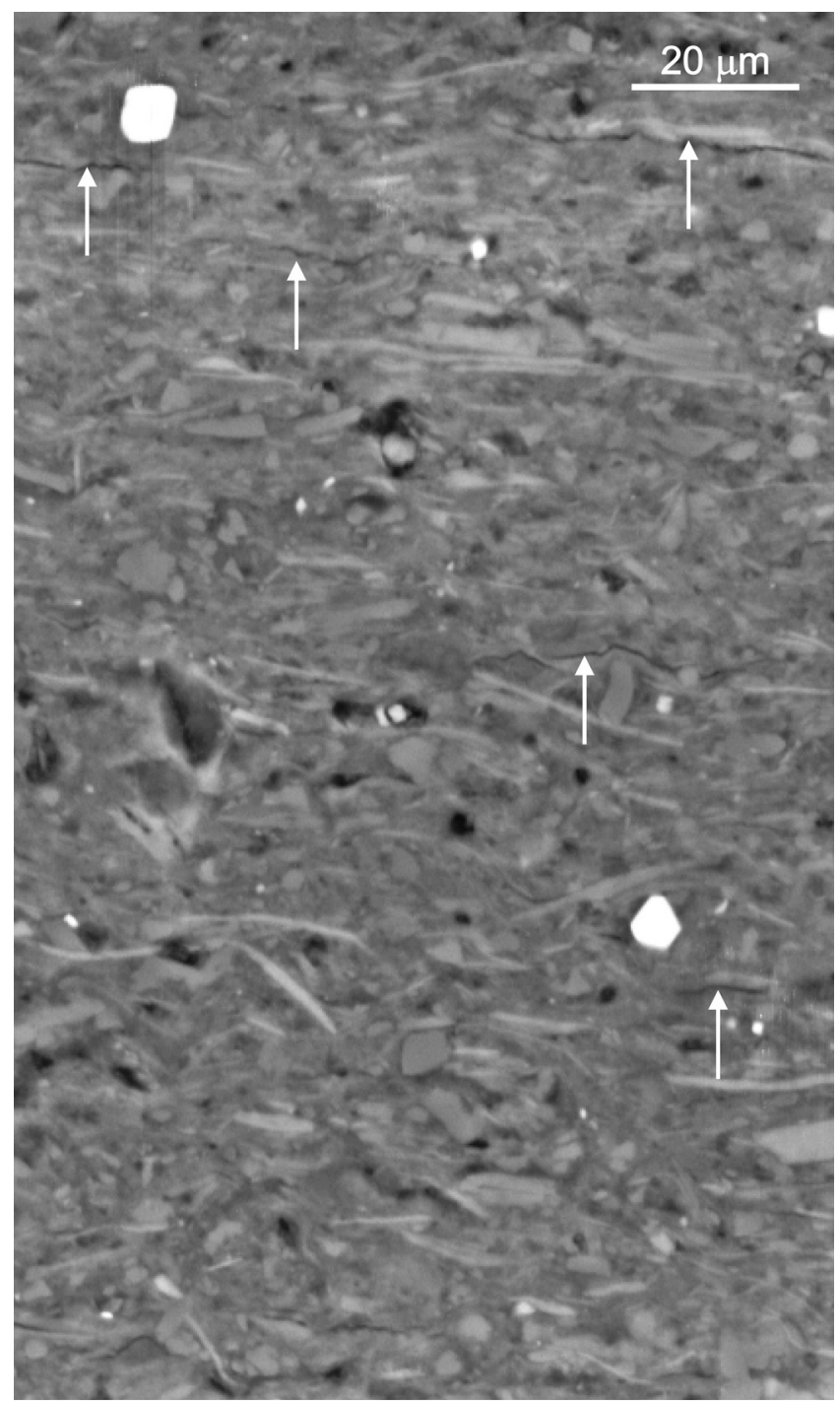

Figure 1. An Officer Basin shale showing particle alignment and the presence of microfractures (white arrows). the most relevant for shale microstructure. The suggested method works quite well for predicting anisotropic elastic moduli of shales on the basis of CPD and silt fraction. However, it fails to predict stress dependency of elastic properties of shales (Pervukhina et al., 2008a, 2008b), which has been observed in many experiments (e.g., Jakobsen and Johansen, 2000; Dewhurst and Siggins, 2006; Delle Piane et al., 2010; Kuila et al., 2011).

The problem of stress dependency of shale properties is important for such applications as pore pressure prediction and time lapse seismic monitoring. Despite this, there is no widely accepted theory for stress dependency of elastic properties of shales. Sayers (1999) studies stress dependencies of air-dry and fluid-saturated shales using the Sayers and Kachanov (1995) excess compliance approach and obtained ratios of normal to tangential compliances of clay platelet contacts for the both cases, assuming the contacts were well aligned. Prioul et al. (2004) and Prioul and Lebrat (2004) describe the stress dependency of shales using a model based on nonlinear elasticity with three stress-sensitive (nonlinear) parameters, although this model is suitable only for a stress range where stiffnesses are quasilinear with stress. Shapiro and Kaselow (2005) suggest a stress dependency model for orthorhombic media based on a dual porosity approach. Their model is based on the bimodal distribution of pore compliances and superposition of deformation fields caused by closure/shape change of these two groups of soft and stiff pores under applied stress. Ciz and Shapiro (2009) use this approach to build a model of experimental shale measurements from the North Sea previously reported by Hornby (1998) and show a good model fit to the measurements. However in the isotropic limit, the model of Ciz and Shapiro (2009) has been shown to be equivalent to the isotropic version of the model of Sayers (1999) with exponential dependency of excess compliances on pressure and with an additional assumption that normal and tangential compliances of each grain contact are equal (Gurevich et al., 2009). This assumption may not be physically adequate, especially for water-saturated rocks at ultrasonic frequencies, because water in intergranular pores and microfractures should strongly reduce normal, but not tangential, compliance.

In this paper, we propose a new model that allows description of stress dependency of all five elastic coefficients of TI shales by treating both the orientation distribution of clay platelets and the compliance ratio of platelet contacts as model parameters. To this end, we combine the dual porosity approach of Shapiro and Kaselow (2005) with the noninteraction approximation of Sayers and Kachanov (1995).

\section{MODELING OF THE EFFECT OF ISOTROPIC STRESS ON THE ANISOTROPIC ORIENTATION OF DISCONTINUITIES}

Following Shapiro (2003), Shapiro and Kaselow (2005), and Ciz and Shapiro (2009), we assume that variation of elastic properties of a shale subjected to effective confining pressures of up to $60 \mathrm{MPa}$ can be explained by closure of soft (compliant) porosity. Soft porosity is a small part of total porosity and consists of pores with high compliances, such as fractures, cracks, and grain or clay platelet contacts. Figure 1 shows a scanning electron microscope image that illustrates existence of both stiff and compliant pores in shales. 
We model shale as an intrinsically TI medium that is permeated with discontinuities such as grain or platelet contacts, cracks, or fractures, which are distributed anisotropically. We assume that the probability density for a particular orientation can be written as

$$
W(\theta, \phi)=\frac{1+\eta \cos ^{2} \theta}{\int_{0}^{2 \pi} \int_{0}^{\pi}\left(1+\eta \cos ^{2} \theta\right) \sin \theta d \theta d \phi}=\frac{1+\eta \cos ^{2} \theta}{4 \pi(1+\eta / 3)},
$$

where $\theta$ is an angle between the $z$-axis and the normal to the crack surface (range $[0, \pi]$ ), $\phi$ determines the rotation about the $z$-axis (range $[0,2 \pi]$ ), and $\eta$ is the crack orientation anisotropy parameter. Isotropic distribution of cracks corresponds to the case when $\eta=0$, and in a case when $\eta$ is large, there is a strong alignment of cracks. One can check that the probability density defined by equation 1 satisfies the normalization condition,

$$
\int_{0}^{2 \pi} \int_{0}^{\pi} W(\theta, \phi) \sin \theta d \theta d \phi=1 .
$$

The exact geometry of individual cracks is not specified. Instead, the behavior of cracks is defined by $B_{T}$, the specific tangential compliance (tangential compliance of a crack with a unit surface area) and a ratio $B=B_{N} / B_{T}$, where $B_{N}$ is specific normal compliance. For parameterization purposes, only effective properties are important, so the surface area $A$ and the compliance ratio $B$ are assumed to be the same for all cracks. We assume that $B_{N}$ and $B_{T}$ are independent of cracks orientation and do not change with stress (for analysis of this assumption for isotropic rocks, see Pervukhina et al. (2010). Following Schoenberg (2002), Shapiro (2003), and Shapiro and Kaselow (2005), we further assume that the surface area of individual cracks decreases with stress exponentially. Therefore, a specific surface area per unit volume $s_{0}=N_{0} A$ also varies exponentially with stress, such that

$$
s(P)=N_{0} A^{0} \exp \left(-P / P_{c}\right)
$$

where $N_{0}$ is the total number of cracks integrated over all angles, $A^{0}$ is the specific surface area of an individual crack at zero stress, $P$ is effective stress, and $P_{c}$ is a characteristic crack closing pressure.

When a rock with this distribution of discontinuities is subjected to a small compressive isotropic stress, the density of cracks along a particular plane is assumed to be reduced exponentially with the normal stress traction acting on that plane. The area of the cracks in the case of their anisotropic distribution will reduce differently in different directions. An effect of this anisotropic variation of the crack areas on elastic properties can be modeled using the Sayers and Kachanov (1995) noninteractive approximation:

$$
\begin{gathered}
\Delta S_{i j k \ell} \equiv S_{i j k \ell}-S_{i j k \ell}^{0} \\
=\frac{1}{4}\left(\delta_{i k} \alpha_{j \ell}+\delta_{i \ell} \alpha_{j k}+\delta_{j k} \alpha_{i \ell}+\delta_{j \ell} \alpha_{i k}\right)+\beta_{i j k \ell} \\
\alpha_{i j}=\frac{1}{V} \sum_{r} B_{T}^{(r)} n_{i}^{(r)} n_{j}^{(r)} A^{(r)}
\end{gathered}
$$

$$
{ }^{\beta} i j k \ell \mid=\frac{1}{V} \sum_{r}\left(B_{N}^{(r)}-B_{T}^{(r)}\right) n_{i}^{(r)} n_{j}^{(r)} n_{k}^{(r)} n_{\ell}^{(r)} A^{(r)},
$$

Here, $\Delta S_{i j k \ell}$ is the excess compliance caused by the presence of compliant cracks, $S_{i j k \ell}^{0}$ are compliances at high stress with all soft cracks closed, $S_{i j k \ell}$ are the compliances at some intermediate stress, $\delta_{i j}$ is the Kronecker delta, $r$ is the number of planar discontinuities with surface area $A^{(r)}, n_{i}^{(r)}$ and $n_{j}^{(r)}$ are $i$ th and $j$ th components of the unit vector that is normal to the surface of the $r$ th grain boundary in volume $V$; and $B_{N}$ and $B_{T}$ are the normal and tangential compliances of an individual crack.

Substituting equations 1 and 3 into equations $4-6$, we obtain variation in compliances due to the closure of discontinuities at different pressures as follows:

$$
\begin{aligned}
& \Delta S_{11} \equiv S_{11}-S_{11}^{0}= \frac{S_{n} B_{T} \exp \left(-P / P_{c}\right)}{105} \\
& \times(14+4 \eta+21 B+3 B \eta), \\
& \Delta S_{33} \equiv S_{33}-S_{33}^{0}= \frac{s_{n} B_{T} \exp \left(-P / P_{c}\right)}{105} \\
& \times(14+6 \eta+21 B+15 B \eta), \\
& \Delta S_{44} \equiv S_{44}-S_{44}^{0}= \frac{s_{n} B_{T} \exp \left(-P / P_{c}\right)}{105} \\
& \times(42+16 \eta+28 B+12 B \eta), \\
& \Delta S_{66} \equiv S_{66}-S_{66}^{0}= \frac{S_{n} B_{T} \exp \left(-P / P_{c}\right)}{105} \\
& \times \times(42+10 \eta+28 B+4 B \eta), \\
& \Delta S_{13} \equiv S_{13}-S_{13}^{0}= \frac{s_{n} B_{T} \exp \left(-P / P_{c}\right)}{105} \\
& \quad \times(7 B+3 B \eta-7-3 \eta) .
\end{aligned}
$$

Here $s_{n}=s /[4 \pi(1+\eta / 3)]$, and $s=N_{0} A^{0}$ is the specific surface area of cracks per unit volume. The detailed derivation of equations 7-11 is given in Appendix 1. Note that shales exhibit TI anisotropy, and five independent elastic compliances $S_{11}^{0}, S_{33}^{0}$, $S_{44}^{0}, S_{66}^{0}$, and $S_{13}^{0}$ are required even at high effect stresses when all compliant cracks are supposed to be closed. Such intrinsic anisotropy is caused by anisotropic individual clay mineral moduli and preferential orientation of domains of aggregated clay minerals. If we assume $B_{N}=B_{T}$, then equations $7-11$ can be simplified as follows:

$$
\begin{gathered}
\Delta S_{11} \equiv S_{11}-S_{11}^{0}=\frac{s_{n} B_{T} \exp \left(-P / P_{c}\right)}{15}(5+\eta), \\
\Delta S_{33} \equiv S_{33}-S_{33}^{0}=\frac{s_{n} B_{T} \exp \left(-P / P_{c}\right)}{15}(5+3 \eta), \\
\Delta S_{44} \equiv S_{44}-S_{44}^{0}=\frac{s_{n} B_{T} \exp \left(-P / P_{c}\right)}{15}(10+4 \eta), \\
\Delta S_{66} \equiv S_{66}-S_{66}^{0}=\frac{2 s_{n} B_{T} \exp \left(-P / P_{c}\right)}{15}(5+\eta),
\end{gathered}
$$




$$
\Delta S_{13} \equiv S_{13}-S_{13}^{0}=0 .
$$

Thus, to parameterize the stress dependency of shales for the general case when $B_{N} \neq B_{T}$ (i.e., equations $7-11$ above), four parameters are necessary, namely $s_{n} B_{T}$, tangential compliance of an individual crack per unit area multiplied by the normalized specific surface area of cracks per unit volume; $B$, the ratio of crack normal to crack tangential compliance; $\eta$, the crack orientation anisotropy parameter that characterizes angular crack distribution; and $P_{c}$, the characteristic pressure at which compliant pores close. For the case of so-called scalar crack approximation, when $B_{N}=B_{T}$, three parameters, $s_{n} B_{T}, \eta$, and $P_{c}$, are required. Note that the parameters $s_{n}$ and $B_{T}$ are coupled and cannot be obtained independently. Hereafter, we refer to $s_{n} B_{T}$ as $B_{T}$, bearing in mind that it is, in fact, multiplied by the normalized specific surface area of cracks per unit volume. Below, we fit experimentally measured stress dependencies of elastic prop-

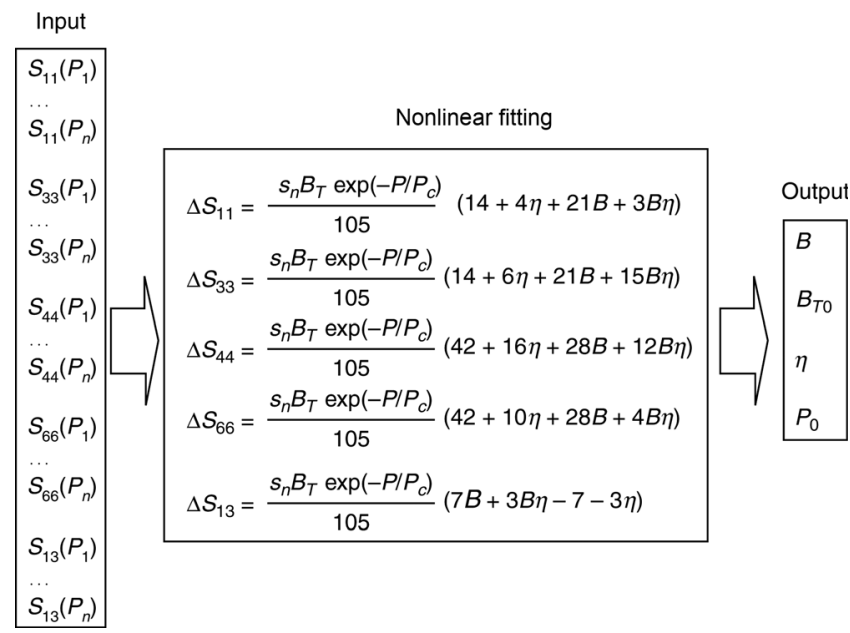

Figure 2. Schematic diagram of fitting procedure. Compliances calculated from experimentally measured velocities at different isotropic effective stresses are fitted using equations 7-11. As a result of the fitting, four fitting parameters are obtained.

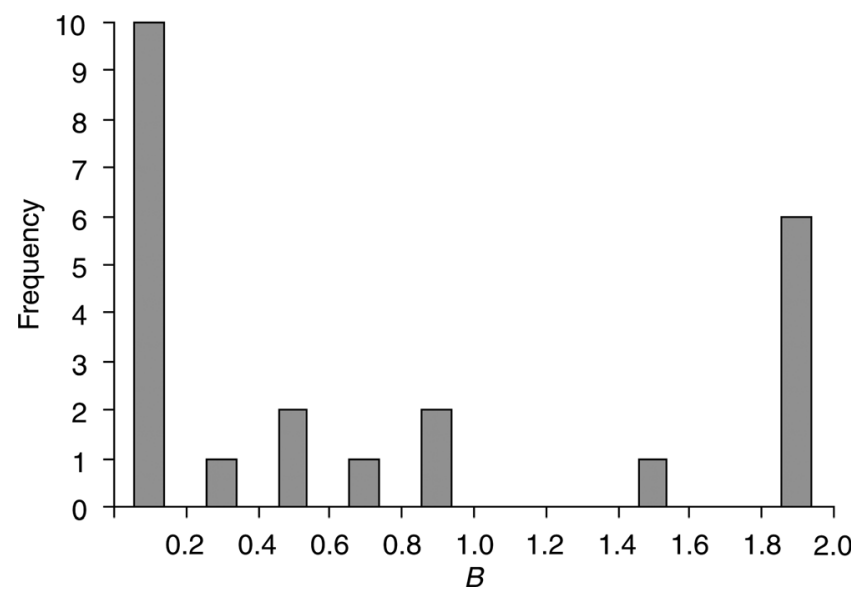

Figure 3. Histogram of the ratio of normal to tangential compliance for all the shale samples. Most of the values are far from unity. erties of shales using both the general set of equations 7-11 and the scalar crack approximation (equations 12-16) and show the uncertainties that are introduced in the latter case.

\section{DATA}

We applied our model to a number of shales from the Officer Basin, Bass Basin, Carnarvon Basin (offshore Australia), Africa, and the North Sea (Pervukhina et al., 2008a). The details of the experimental procedure and sample preparation can be found in Dewhurst and Siggins (2006) and Dewhurst et al. (2008a, 2008b). We also used stress dependencies measured by Wang (2002) on shale samples from Africa, North Sea shales, Gulf Coast shale, and hard shales. We used measurements obtained for samples cut parallel and perpendicular to bedding, as application of an isotropic stress would not change the TI symmetry of these samples. All the samples were preserved (prevented from drying) before measurements. All the data were obtained in experiments with controlled pore pressure. The measurements for shales from the Officer Basin, Bass Basin, Carnarvon Basin, Africa, and the North Sea were obtained under a pore pressure of $5 \mathrm{MPa}$. The pore pressures for other shales are given in Wang (2002).

The shale samples were recovered from depths between 200 and $3604 \mathrm{~m}$ and vary in their physical properties and in their mineralogy. Unfortunately, Wang (2002) does not report information about overburden pressure, diagenesis, geological history, clay content, or mineralogy of the investigated shales. The depth of origin of the shale samples is the only environmental parameter known for all the shales.

\section{FITTING PROCEDURE AND TRENDS IN MICROCRACK PROPERTIES}

The fitting procedure is schematically shown in Figure 2. Compliances calculated from experimentally measured velocities at different isotropic effective stresses are fitted using equations 7-11 or 12-16. The Levenberg-Marquardt method (Moré, 1977) is used for nonlinear fitting of experimentally obtained stress dependencies of the elastic compliances of shales with equations $7-11$ or $12-16$, considering $B_{T}, B, P_{c}$, and $\eta$ as fitting parameters. We first fit the data using the scalar crack approximation in equations $12-16$ and then use the resultant parameters $B_{T}, P_{c}$, and $\eta$ and $B=1$ as an initial estimate for the general set of equations $7-11$. For the second procedure, we use a condition $B$ $\leq 2$ as a constraint to fit the results.

A histogram of compliance ratio $B$, obtained using equations $7-11$, is shown in Figure 3. The values are distributed in three distinct groups: (1) small values less than 0.2 , (2) normal values from 0.7 to 1.1 , and (3) large values of about 2. Departure from unity is observed even in the group with the values from 0.7 to 1.1. This suggests that the ratio $B=1$, which corresponds to the model of Ciz and Shapiro (2009), may not be adequate for shales.

To understand the excess in misfit of the experimental stress dependencies caused by the assumption that $B=1$, we compare the results of the experimental data fitting using equations 7-11 and 12-16. The misfits are shown in Figure 4a by solid dots (equations 7-11) and open circles (equations 12-16). Figure 4b shows the relative excess of misfit caused by the assumption 
that $B_{N}=B_{T}$. The relative misfits are close to zero, and consequently the misfits are almost the same when $B$ is close to unity. However, in other cases, the misfit by equation set 12-16 noticeably exceeds the one obtained by use of equations 7-11 (note that the plot in Figure $4 \mathrm{a}$ is in logarithmic scale). The error caused by use of a scalar crack approximation (equations 12-16) exceeds the error resulted from fitting with the full set of equations $7-11$ by up to $70 \%$. Similar results are obtained by Angus et al. (2009), who used a synthetic data set to estimate an error caused by usage of a scalar crack approximation instead of the full set of equations. From here onward, we use only equations $7-11$ to fit the experimental stress dependencies of elastic properties of shales to avoid additional errors caused by the assumption that $B_{N}=B_{T}$.

All four fitting parameters are plotted versus depth of shale extraction in Figure 5. Crack orientation anisotropy parameter $\eta$ shows a general linear growth with depth of origin (Figure 5a), although there is significant scatter of the fitting values. Higher values of $\eta$ mean better alignment of the cracks in the bedding plane; consequently, an increase of $\eta$ with the depth indicates increased alignment of discontinuities with increasing overburden pressure.

Tangential compliance of a single crack normalized to the area of the crack exponentially decreases with depth (Figure $5 b$ ). This implies that cracks are stiffer in shales that are recovered from greater depths than in the shales extracted from shallower depths.

The ratio of normal to tangential compliance initially decreases from 1 to 0.1 with the increase of depth from 1000 to $2500 \mathrm{~m}$ (Figure 5c), implying that normal stiffness of shales grows faster with the overburden pressure than the tangential one. Then $B$ increases again, reaching 2 at a depth of $3500 \mathrm{~m}$. In other words, in the depth range of 1000-2500 m, cracks become relatively stiffer in the plane of the crack than in the normal direction with increasing depth. However, below $2500 \mathrm{~m}$, the reverse seems to occur. Although it is commonly accepted that individual shales can become stiffer and more anisotropic with depth, this variation of the ratio of normal to tangential compliance is a new finding that has yet to be explained.

The characteristic pressure $P_{c}$ shows no obvious trend with the depth of origin (Figure 5d). The characteristic pressure is equal to $20 \mathrm{MPa}$ for the shallow (less than $1500 \mathrm{~m}$ ) and deep (more than $2500 \mathrm{~m}$ ) depths. For the intermediate depth of 1500 $2500 \mathrm{~m}, P_{c}$ drops to $10 \mathrm{MPa}$.

\section{DISCUSSION}

Our model of pressure dependency of shale properties can be compared to that of Ciz and Shapiro (2009). Although our fits to experimental data are somewhat better, this is achieved by introducing a new parameter, the compliance ratio $B$. Is this justified? Connected to this, we note that in the isotropic limit, the equations of Ciz and Shapiro (2009) reduce to a particular case of the equations of Shapiro (2003) with the ratio of bulk to shear piezosensitivities $(Q)$ equal to $(1+v) /(1-2 v)$, where $v$ is Poisson's ratio at the high-pressure limit where all compliant porosity is closed. In turn, it has been shown (Gurevich et al., 2009) that equations of Shapiro (2003) (without dependency of stiff porosity on pressure) are equivalent to an isotropic version of the Sayers and Kachanov (1995) equations with exponential dependency of both $B_{N}$ and $B_{T}$ on pressure. There is also a direct relationship between $Q$ and the compliance ratio $B=B_{N} / B_{T}$ in the isotropic version of the Sayers-Kachanov model. In particular, $Q=(1+v) /(1-2 v)$ corresponds to $B=1$ (Gurevich et al., 2009, equation 10). Although improvement of the fit by allowing $B$ to be a free parameter may not be huge, we think that assumption $B=1$ may not be physically adequate, especially for water-saturated rocks at ultrasonic frequencies, because water in intergranular microcracks should strongly reduce $B_{N}$ but not $B_{T}$. For isotropic rocks, this has been confirmed by analysis of laboratory measurements (see Sayers and Han, 2002, and Angus et al., 2009). We believe this is also logical for anisotropic rocks, including shales, even if quality of shale data may not be sufficient at present to show this conclusively. We also note that even with a new parameter $B$, our model still has fewer parameters than the model of Ciz and Shapiro (2009).

The developed model allows one to obtain statistically qualitative properties of microcracks in shales. For the data set analyzed, these properties show moderate to good correlations with the depth of extraction. Even though some of the parameters exhibit only a broad correlation with the depth of origin, such general correlations for shales may be helpful for initial characterization of stress dependencies of elastic properties of shales in new basins. Thus, we believe that more extensive and statistically representative studies are required to confirm, improve (if possible), and understand the physical meaning of the obtained broad correlations. For example, it is well known that stress history and temperature also control mechanical and elastic

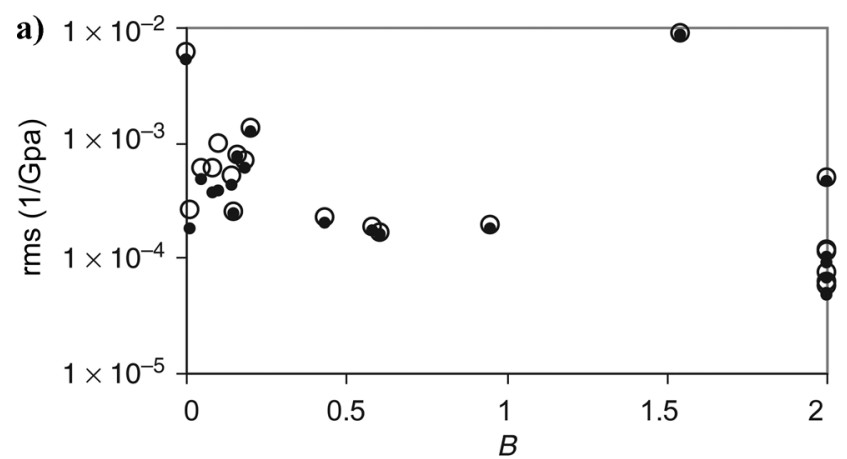

b)

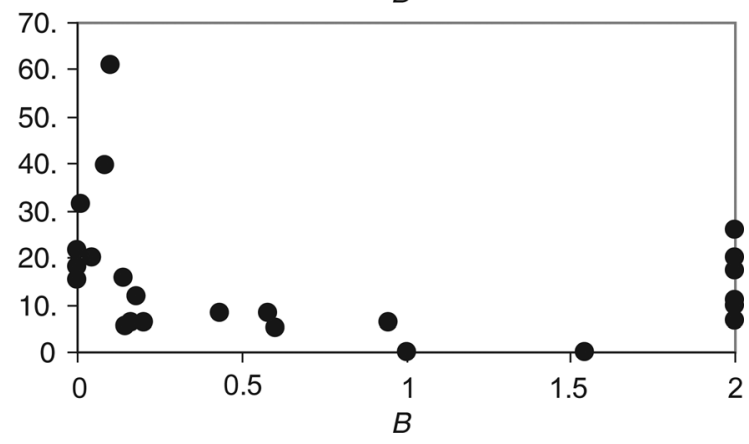

Figure 4. Quality of fitting of the experimental stress dependencies of elastic coefficients. (a) Misfits from equations 7-11 and equations $12-16$ are shown by solid dots and open circles, respectively. (b) Relative excess in misfit caused by use of equations 12-16. 
properties of shales (e.g., Nygard et al., 2004; Peltonen et al., 2009) through poroelastic effects and diagenetic mineral reactions, respectively. Unfortunately, such data were not available for most of the shales evaluated, so we were unable to characterize the shales more specifically in these terms at this stage. However, even with this restriction, the model gives some results that seem geologically intuitive, suggesting a physically sound basis for further development.

From analyzing this limited data set, one can conclude that the crack orientation anisotropy parameter $\eta$ shows only a broad correlation with the depth. Although clay particle orientation and associated microfractures are often seen as dependent on overburden stress, this can also occur through other less well defined processes that are not governed by depth alone. In addition, clay alignment also can depend on the volume of the silt fraction and the shape of silty grains, which may result in differential compaction of clays and wrapping around more rigid particles. Hence, larger data sets may not drastically improve the correlation between $\eta$ and depth of extraction or overburden pressure.

Shear compliance $\left(B_{T}\right)$ shows a good correlation with depth of extraction (Figure 5b). More complex behavior is observed for the ratio of normal to tangential compliance $B$ (Figure $5 \mathrm{c}$ ) in that it decreases from 1 to 0.1 between 1000 and $2500 \mathrm{~m}$ and then increases above unity to $3600 \mathrm{~m}$. This may reflect the effects of diagenesis that generally starts at a depth of about 2000-3000 m, driven by temperatures above $65^{\circ} \mathrm{C}$ (Ruud et al., 2003; Avseth et al., 2008; Storvoll and Brevik, 2008). It should be noted that the ratio of normal to tangential compliance in a given rock will reflect the roughness of the crack surfaces, and this might be affected by mineralization. The values of $B$ estimated for real rocks often exceed theoretical predictions for traction-free cracks (which always yields $B<1$ ). In particular, Angus et al. (2009) observes values of $B_{N} / B_{T}$ up to 1.75 for dry shales. MacBeth and Schuett (2007) find that the ratio $B$ may increase to the values above unity for thermally damaged samples. Possible contact mechanisms and the effect of mineralization on the values of $B$ are discussed in detail in Sayers et al. (2009) and Kachanov et al. (2010). The characteristic stress $P_{c}$ also shows a minimum in absolute values at depths of $\sim 2500 \mathrm{~m}$, similar to that observed for $B$; this may support our speculative suggestion that the microcrack parameters reflect the effects of mechanical compaction and diagenesis undergone by a particular shale sample. More data and detailed information on shale mineralogy and microstructure are required to test these observations.

The model we have developed can also be used for predicting the stress dependency of unknown elastic parameters from the known ones. This problem is practically important both for laboratory measurements in shales, where the $c_{13}$ coefficient is often unreliable, and for field data analysis, where log data allow determination of only four of five elastic coefficients of TI media (Sinha et al., 2006). Note that the fitting problem described by equations 7-11 is overdetermined (see Figure 2). Elastic compliances used for the fitting are calculated for each effective stress at which ultrasonic velocities were measured. For instance, if ultrasonic velocities were measured at $n_{p}$ effective stresses, we have $5 n_{p}$ equations in total. Thus, the four fitting parameters might be determined from experimental stress dependencies of an incomplete set of elastic coefficients. If, for example, compliances $S_{11}, S_{33}, S_{44}$, and $S_{66}$ are known at four different effective stresses, we have a total of 16 equations. The problem is still over determined in that four output parameters that provide the best fit of our experimental data can be found and then used to calculate the variation of $S_{13}$ at different effective stresses using equation 11 . If we assume that only $S_{11}, S_{33}$, and $S_{66}$ compliances are known at four different effective stresses, we obtain a system of 12 equations that still allows solutions for the four parameters $B, B_{T 0}, \eta$, and $P_{c}$. Then, the variations of elastic compliances $S_{13}$ and $S_{44}$ can be found with equations 9 and 11. For sample G3 from brine-saturated hard shales (Wang, 2002), Figure 6 shows the prediction of stress dependencies of the elastic coefficients, Thomsen anisotropy parameters, and the anellipticity parameter (Tsvankin, 1997) using as input stress dependencies of five, four, and three elastic coefficients. The fitting parameters $\eta, B, s B_{T}$, and $P_{c}$ are 20, 2, and 0.007 $\mathrm{GPa}^{-1}$ and $20 \mathrm{MPa}$, respectively. The predictions obtained from input of four elastic coefficients $\left(S_{11}, S_{33}, S_{44}, S_{66}\right)$ are almost indistinguishable from those obtained for the input of five; the differences for the case of input of three elastic coefficients $\left(S_{11}, S_{33}, S_{66}\right)$ are also small. However, it is important to note explicitly that this prediction does not include prediction of the intrinsic compliances $S_{i j}^{0}$ or of the stress dependence due to the hard compliance at large effective stress.
Figure 5. Variations with depth of (a) crack orientation distribution $\theta$, (b) tangential compliance $S B_{T}$, (c) characteristic pressure $P_{0}$, (d) ratio of normal to tangential compliance $B$. 

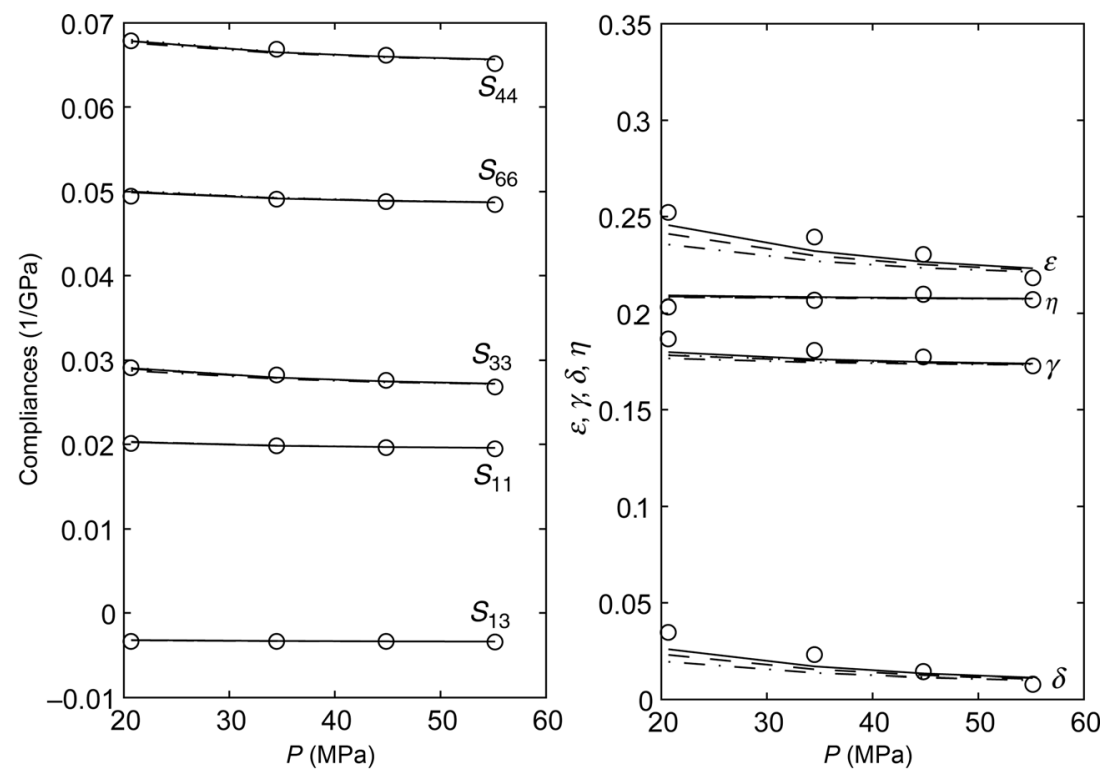

Figure 6. Compliances (left) and anisotropy parameters (right) for both measurements and our model on brine-saturated hard shale (sample G3 from Wang, 2002). Values calculated from ultrasonic measurements are shown by circles. Fits using the full set of five compliances are shown by thick lines. Thin lines show fits using incomplete sets of compliances. In most cases, the thin and thick lines coincide.

\section{CONCLUSIONS}

A new stress dependency model for TI media was developed and used to parameterize stress dependencies of the elastic properties of about 20 shales. The four fitting parameters (namely, specific tangential compliance of a single discontinuity, ratio of normal to tangential compliances, characteristic pressure, and crack orientation anisotropy parameter) show moderate correlations with the depth from which the shale was extracted. With increasing depth, the tangential compliance exponentially decreases. The crack orientation anisotropy parameter broadly increases with the depth for most of the shales, indicating that cracks are becoming more aligned. The ratio of normal to shear compliance and the characteristic pressure decrease to depths of $2500 \mathrm{~m}$ and then increase below this to $3600 \mathrm{~m}$. The suggested model also allows the prediction of stress dependency of all five elastic compliances if only three or four compliances are known. This could be useful for the reconstruction of stress dependencies of all five elastic compliances of shale from log data, for example.

\section{ACKNOWLEDGMENTS}

The authors are grateful to Romain Prioul, Serge Shapiro, Andrej Bona, Tamas Nemeth, and an anonymous reviewer for useful advice that helped drastically improve the paper. Joel Sarout is gratefully thanked for valuable discussions.

\section{APPENDIX A}

\section{STRESS DEPENDENCY OF ELASTIC COMPLIANCES OF TI MEDIA WITH ANISOTROPICALLY DISTRIBUTED DISCONTINUITIES}

Orientation distribution of cracks is commonly described with the help of two coordinate systems, global (measurement system) and local (associated with the orientation of an individual crack). The two systems are related by three Eulerian angles $(\theta, \phi, \psi)$, where $\theta$ ranges lie in the range $[0, \pi]$ and determine the angle between the $z^{\prime}$-axis of the local system and the $z$-axis of global system, $\phi$ controls the rotation about the $z$-axis (range $[0,2 \pi]$ ), and $\psi$ specifies the rotation of the crack about the local $z^{\prime}$-axis (range $[0,2 \pi]$ ). If the $z^{\prime}$-axis of the local coordinate system coincides with the normal to the crack surface, we reduce the number of angles to two, namely, $\theta$ and $\phi$. The spherical system determined with these two angles and the $z$-axis, which here is assumed to coincide with the axis of rotational symmetry of the medium, is a convenient system for calculating $\alpha_{i j}$ and $\beta_{i j k l}$ tensors from equations 5 and 6 . Note that the three components of a unit vector in a spherical coordinate system can be written as

$$
\begin{gathered}
n_{1}=\sin \theta \cos \phi, \\
n_{2}=\sin \theta \sin \phi, \\
n_{3}=\cos \theta .
\end{gathered}
$$

Calculation of $\alpha_{i j}$ and $\beta_{i j k \ell}$ tensors for cracks with a particular orientation distribution function, determined by equations 1 and 2 and with surface area governed by equation 3 , involves integration over all possible orientations of cracks, i.e., over all angles $\theta$ and $\phi$ that define the direction of the normal to a crack surface. Integration over the angles $\theta$ and $\phi$ is an integration over the surface of a unit sphere; the infinitesimal element of the surface should be written as $\sin \theta d \theta d \phi$.

The probability that the normal to the surface of the crack forms an angle $\theta$ with the $z$-axis that lies in the interval $\left[\theta_{1}, \theta_{2}\right]$ can be written as

$$
P\left(\theta_{1} \leq \theta \leq \theta_{2}\right)=\int_{0}^{2 \pi} \int_{0}^{\pi} W(\theta, \phi) \sin \theta d \theta d \phi
$$


or, if we take into account equation 1, as follows:

$$
\begin{aligned}
P\left(\theta_{1} \leq \theta \leq \theta_{2}\right) & =\int_{0}^{2 \pi} \int_{\theta_{1}}^{\theta_{2}} \frac{1+\eta \cos ^{2} \theta}{4 \pi(1+\eta / 3)} \sin \theta d \theta d \phi \\
& =\frac{1}{4 \pi(1+\eta / 3)} \int_{0}^{2 \pi} \int_{\theta_{1}}^{\theta_{2}}\left(1+\eta \cos ^{2} \theta\right) \sin \theta d \theta .
\end{aligned}
$$

The specific number of cracks per unit volume whose normals form an angle $\theta$ with the $z$-axis that lie in the interval $\left[\theta_{1}, \theta_{2}\right]$ can then be written as

$$
\begin{aligned}
N\left(\theta_{1} \leq \theta \leq \theta_{2}\right)= & \frac{N_{0}}{4 \pi(1+\eta / 3)} \\
& \times \int_{0}^{2 \pi} \int_{\theta_{1}}^{\theta_{2}}\left(1+\eta \cos ^{2} \theta\right) \sin \theta d \theta d \phi
\end{aligned}
$$

where $N_{0}$ is the total number of cracks.

In equation 5 , summation over all cracks can be replaced with integration over all angles, and $\alpha_{i j}$ for a unit volume can be written as

$$
\alpha_{i j}=\frac{N_{0}}{4 \pi(1+\eta / 3)} \int_{0}^{2 \pi} \int_{0}^{\pi} B_{T} A\left(1+\eta \cos ^{2} \theta\right) n_{i} n_{j} \sin \theta d \theta d \phi .
$$

Taking into account equation 3 and assuming that $B_{T}$ is the same for all crack orientations, equation A-7 can be rewritten as:

$$
\alpha_{i j}=\frac{N_{0} B_{T} A^{0} \exp \left(-P / P_{c}\right)}{4 \pi(1+\eta / 3)} \int_{0}^{2 \pi} \int_{0}^{\pi}\left(1+\eta \cos ^{2} \theta\right) n_{i} n_{j} \sin \theta d \theta d \phi .
$$

Noting that the specific surface area of cracks per unit volume is equal to $s_{0}=N_{0} A^{0}$ and denoting $s_{n}=s_{0} /(1+\eta / 3)$, we can rewrite equation A-8 as follows:

$$
\alpha_{i j}=\frac{s_{n} B_{T} \exp \left(-P / P_{c}\right)}{4 \pi} \int_{0}^{2 \pi} \int_{0}^{\pi}\left(1+\eta \cos ^{2} \theta\right) n_{i} n_{j} \sin \theta d \theta d \phi .
$$

Taking into account equations A-1-A-3 and integrating over all angles in equation A-9 we finally get the tensor $\alpha_{i j}$ as follows:

$$
\alpha_{i j}=\frac{1}{15} s_{n} B_{T} e^{-\frac{P}{P_{c}}}\left[\begin{array}{ccc}
5+\eta & 0 & 0 \\
0 & 5+\eta & 0 \\
0 & 0 & 5+3 \eta
\end{array}\right]
$$

The tensor $\beta_{i j k \ell}$ for a unit volume can be obtained in a similar way:

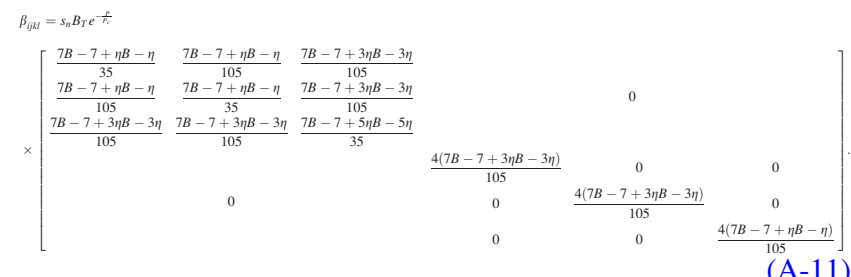

(A-11)

Substituting $\alpha_{i j}$ and $\beta_{i j k \ell}$ into equation 4 , we obtain the excess compliances (equations 7-11) caused by cracks with an orientation distribution function given by equation 1 . If $B=1$, equations 7-11 reduce to equations $12-16$.

\section{REFERENCES}

Aleksandrov, K. S., and T. V. Ryzhova, 1961, Elastic properties of rockforming minerals II: Layered silicates: Bulletin of the Academy of Sciences of the USSR Geophysics Series, English translation, 12, 11651168 .

Angus, D. A., J. P. Verdon, Q. J. Fisher, and J. M. Kendall, 2009, Exploring trends in microcrack properties of sedimentary rocks: An audit of dry-core velocity-stress measurements: Geophysics, 74, no. 5, E193E203, doi:10.1190/1.3183940.

Avseth, P., A. Draege, A.-J. van Wijngaarden, T. A. Johansen, and A. Jørstad, 2008, Shale rock physics and implications for AVO analysis: A North Sea demonstration: The Leading Edge, 27, 788-797, doi:10.1190/1.2944166.

Banik, N. C., 1984, Velocity anisotropy in shales and depth estimation in the North Sea basin: Geophysics, 49, 1411-1419, doi:10.1190/1.1441770.

Bayuk, I. O., M. Ammerman, and E. M. Chesnokov, 2007, Elastic moduli of anisotropic clay: Geophysics, 72, no. 5, D107-D117, doi:10.1190/ 1.2757624 .

Castagna, J. P., M. 1. Batzle, and T. K. Kan, 1993, Rock physics — The link between rock properties and AVO response in offset-dependent reflectivity, in J. P. Castagna and M. Backus, eds., Theory and practice of AVO analysis: SEG, 135-171.

Ciz, R., and S. A. Shapiro, 2009, Stress-dependent anisotropy in transversely isotropic rocks: Comparison between theory and laboratory experiment on shale: Geophysics, 74, no. 1, D7-D12, doi:10.1190/1.3008546.

Delle Piane, C., D. N. Dewhurst, A. F. Siggins, and M. D. Raven, 2010, Stress-induced anisotropy in brine saturated shale: Geophysical Journal International, 184, 897-906, doi:10.1111/j.1365-246X.2010. 04885.x.

Dewhurst, D. N., and A. F. Siggins, 2006, Impact of fabric, misrocracks and stress field on shale anisotropy: Geophysical Journal International, 165, 135-148, doi:10.1111/j.1365-246X.2006.02834.x.

Dewhurst, D. N., A. F. Siggins, U. Kuila, M. B. Clennell, M. D. Raven, and H. M. Nordgård-Bolås, 2008a, Elastic, geomechanical and petrophysical properties of shales: Presented at the American Rock Mechanics Association Symposium.

, 2008b, Rock physics, geomechanics and rock properties in shales: Where are the links?: Presented at the 1st Southern Hemisphere International Rock Mechanics Symposium.

Gurevich, B., D. Makarynska, and M. Pervukhina, 2009, Are pennyshaped cracks a good model for compliant porosity?: 79th Annual International Meeting, SEG, Expanded Abstracts, 3431-3434.

Han, D.-H., A. Nur, and D. Morgan, 1986, Effects of porosity and clay content on wave velocities in sandstones: Geophysics, 51, 2093-2107, doi:10.1190/1.1442062.

Hornby, B. E., 1998, Experimental laboratory determination of the dynamic elastic properties of wet, drained shales: Journal of Geophysical Research, 103, B12, 29945-29964, doi:10.1029/97JB02380.

Hornby, B. E., L. M. Schwartz, and J. A. Hudson, 1994, Anisotropic effective-medium modeling of the elastic properties of shales: Geophysics, 59, 1570-1583, doi:10.1190/1.1443546.

Jakobsen, M., and T. A. Johansen, 2000, Anisotropic approximations for mudrocks: A seismic laboratory study: Geophysics, 65, 1711-1725, doi:10.1190/1.1444856.

Johansen, T. A., B. O. Ruud, and M. Jakobsen (2004), Effect of grain scale alignment on seismic anisotropy and reflectivity of shales: Geophysical Prospecting, 52, 133-149.

Johnston, J. E., and N. I. Christensen, 1995, Seismic anisotropy of shales: Journal of Geophysical Research, 100, B4, 5991-6003, doi:10.1029/ 95JB00031.

Kachanov, M., R. Prioul, and J. Jocker, 2010, Incremental linear-elastic response of rocks containing multiple rough fractures: Similarities and 
differences with traction-free cracks: Geophysics, 75, no. 1, D1-D11, doi:10.1190/1.3268034

Katahara, K. W., 1996, Clay mineral elastic properties: 66th Annual International Meeting, SEG, Expanded Abstracts, 1691-1694.

Kuila, U., D. N. Dewhurst, A. F. Siggins, and M. D. Raven, 2010, Stress anisotropy and velocity anisotropy in low porosity shale: Tectonophysics, doi:10.1016/j.tecto.2010.09.023.

MacBeth, C., and H. Schuett, 2007, The stress dependent elastic properties of thermally induced microfractures in aeolian Rotliegend sandstone: Geophysical Prospecting, 55, no. 3, 323-332, doi:10.1111/j.13652478.2007.00601.x

Moré, J. J., 1977, The Levenberg-Marquardt algorithm: Implementation and theory: Springer Verlag.

Nygard, R., M. Gutierrez, R. Gautam, and K. Høeg, 2004, Compaction behavior of argillaceous sediments as function of diagenesis: Marine and Petroleum Geology, 21, 349-362, doi:10.1016/j.marpetgeo.2004 01.002 .

Ortega, J. A., F.-J. Ulm, and Y. Abousleiman, 2007, The effect of the nanogranular nature of shale on their poroelastic behavior: Acta Geotechnica, 2, no. 3, 155-182

Peltonen, C., O. Marcussen, K. Bjørlykke, and J. Jahren, 2009, Clay mineral diagenesis and quartz cementation in mudstones: The effects of the smectite to illite reaction on rock properties: Marine and Petroleum Geology, 26, 887-898, doi:10.1016/j.marpetgeo.2008.01.021.

Pervukhina, M., D. Dewhurst, B. Gurevich, U. Kuila, A. F. Siggins, M Raven, and H. M. Nordgărd Bolås, 2008a, Stress-dependent elastic properties of shales: Measurement and modeling: The Leading Edge, 27, 772-779, doi:10.1190/1.2944164.

Pervukhina, M., D. N. Dewhurst, U. Kuila, A. F. Siggins, and B. Gurevich, 2008b, Stress dependent anisotropy in shales: Measurements and modelling: Presented at the 1st Southern Hemisphere International Rock Mechanics Symposium.

Pervukhina, M., B. Gurevich, D. N. Dewhurst, and A. F. Siggins, 2010, Experimental verification of the physical nature of velocity-stress relationship for isotropic porous rocks: Geophysical Journal International, doi:10.1111/j.1365-246X.2010.04535.x.

Prioul, R., A. Bakulin, and V. Bakulin, 2004, Nonlinear rock physics model for estimation of 3D subsurface stress in anisotropic formations: Theory and laboratory verification: Geophysics, 69, 415-425, doi:10.1190/1.1707061.

Prioul, R., and T. Lebrat, 2004, Calibration of velocity-stress relationships under hydrostatic stress for their use under non-hydrostatic stress conditions: 74th Annual International Meeting, SEG, Expanded Abstracts, 1698-1701.

Ruud, B. O., M. Jakobsen, and T. A. Johansen, 2003, Seismic properties of shales during compaction: 73rd Annual International Meeting, SEG, Expanded Abstracts, 1294-1297.
Sayers, C., 1999, Stress-dependent seismic anisotropy of shales: Geophysics, 64, 93-98, doi:10.1190/1.1444535.

, 2005, Seismic anisotropy of shales: Geophysical Prospecting, 53, 667-676, doi:10.1111/j.1365-2478.2005.00495.x.

Sayers, C., and D. -H. Han, 2002, The effect of pore fluid on the stress-dependent elastic wave velocities in sandstones: $72^{\text {nd }}$ Annual International Meeting, SEG, Expanded Abstracts, 1842-1845.

Sayers, C., and M. Kachanov, 1995, Microcrack-indused elastic wave anisotropy of brittle rock: Journal of Geophysical Research, 100, B3, 4149-4156, doi:10.1029/94JB03134.

Sayers, C. M., A. D. Taleghani, and J. Adachi, 2009, The effect of mineralization on the ratio of normal to tangential compliance of fractures: Geophysical Prospecting, 57, 439-446, doi:10.1111/j.1365-2478.2008. 00746.x.

Schoenberg, M., 2002, Time-dependent anisotropy induced by pore pressure variation in fractured rock: Journal of Seismic Exploration 11, no. $1-2,83-105$.

Shapiro, S. A., 2003, Elastic piezosensitivity of porous and fractured rocks: Geophysics, 68, 482-486, doi:10.1190/1.1567216.

Shapiro, S. A., and A. Kaselow, 2005, Porosity and elastic anisotropy of rocks under tectonic-stress and pore-pressure changes: Geophysics, 70, no. 5, N27-N38, doi:10.1190/1.2073884.

Sinha, B. K., B. Vissapragada, L. Renlie, and S. Tysse, 2006, Radial profiling of the three formation shear moduli and its application to well completions: Geophysics, 71, no. 6, E65-E77, doi:10.1190/1.2335879.

Storvoll, V., and I. Brevik, 2008, Identifying time, temperature, and mineralogical effects on chemical compaction in shales by rock physics relations: The Leading Edge, 27, 750-756, doi:10.1190/1.2944162.

Thomsen, L., 1986, Weak elastic anisotropy: Geophysics, 51, 1954-1966, doi: $10.1190 / 1.1442051$

Tsvankin, I., 1997, Anisotropic parameters and P-wave velocities for orthorhombic media: Geophysics, 62, 1292-1309, doi:10.1190/1.1444231.

Tsvankin, I., K. Larner, J. Gaiser, and V. Grechka, 2009, Seismic anisotropy: Introduction: Geophysics, 74, no. 5, WB1-WB2, doi:10.1190/ 1.3205035 .

Ulm, F. J., and Y. Abousleiman, 2006, The nanogranular nature of shale: Acta Geotechnica, 1, no. 2, 77-88, doi:10.1007/s11440-006-0009-5

Vanorio, T., M. Prasad, and A. Nur, 2003, Elastic properties of dry clay mineral aggregates, suspensions and sandstones: Geophysical Journal International, 155, 319-326, doi:10.1046/j.1365-246X.2003. 02046.x.

Wang, Z., 2002, Seismic anisotropy in sedimentary rocks, part 2: Laboratory data: Geophysics, 67, 1423-1440, doi:10.1190/1.1512743.

Woeber, A. F., S. Katz, and T. J. Ahrens, 1963, Elasticity of selected rock and minerals: Geophysics, 28, 658-663, doi:10.1190/1.1439242. 